\title{
Modeling of the Electromagnetic Wavefront Sharpening in a Nonlinear Dielectric
}

\author{
M. M. Rezinkina ${ }^{a}$ and O. L. Rezinkin ${ }^{b *}$ \\ ${ }^{a}$ Science and Technology Center of Magnetism of Technical Objects, National Academy of Sciences of Ukraine, \\ Industrial'naya ul. 19, Kharkov, 61106 Ukraine \\ ${ }^{b}$ National Technical University Kharkov Polytechnic Institute, ul. Frunze 21, Kharkov, 61002 Ukraine \\ *e-mail: olegrezinkin@rambler.ru \\ Received November 17, 2009; in final form, June 29, 2010
}

\begin{abstract}
The results of physical and numerical modeling of electromagnetic wavefront sharpening in a nonlinear dielectric are given. A technique of mathematical modeling of wave propagation is described in terms of a modified vector magnetic potential. It is shown how the level of the applied field strength and the degree of nonlinearity of the dielectric affect the electromagnetic wavefront sharpening.
\end{abstract}

DOI: $10.1134 / \mathrm{S} 1063784211030169$

\section{INTRODUCTION}

Short high-power electromagnetic pulses are applied in plasma physics as power sources for lasers and pulsed accelerators, for obtaining high-power electromagnetic radiation, in radiolocation, and also in other fields of science and technology [1]. One of the promising methods for obtaining high-power electromagnetic pulses with a short rise time is the formation of shock electromagnetic waves in a medium with nonlinear electric or magnetic properties [1, 2]. In some cases, direct generation of pulses with steep fronts of current and voltage by switching the capacitive or inductive energy storages to the load is impossible.

The pulses with a short front (on the order of nanoseconds and less) can be obtained with the help of additional sharpening of the electromagnetic wave that has already been generated in double transmission lines (Fig. 1) consisting of potential (3) and earthed (2) plates (extended conductors), between which a working substance (1), i.e., ferroelectric, is located [1, 2]. Application of materials with nonlinear permittivity as a working substance for such forming lines (FLs) [3] has a number of advantages as compared to generation of electromagnetic pulses in the nonlinear medium of a ferromagnet [4]. Since a wave impedance of the lines on nonlinear dielectrics is by many orders lower than in the cases when nonlinear magnetic materials are used, they make it possible to reach significantly higher levels of pulse power and to ensure operation with a low-resistance load.

To generate shock electromagnetic waves of megaand gigawatt power, it is necessary to design a doublewire forming line filled with a nonlinear working medium and operating at high voltages. To ensure the electric strength of the forming line at a voltage on the order of units or tens of kilovolts, the thickness of its working medium for the available nonlinear dielectrics must be no less than several millimeters. Ceramics based on solid solutions of ferroelectrics [5] can be used as the FL working medium. Production of sufficiently thick items from ferroelectric ceramics without cracks and other inhomogeneities reducing the electric strength is a complicated and labor-consuming task. Therefore, in selecting the geometry of the line generating high-voltage shock waves, it is expedient to carry out physical and mathematical simulation of the transient processes arising in this case. The results of

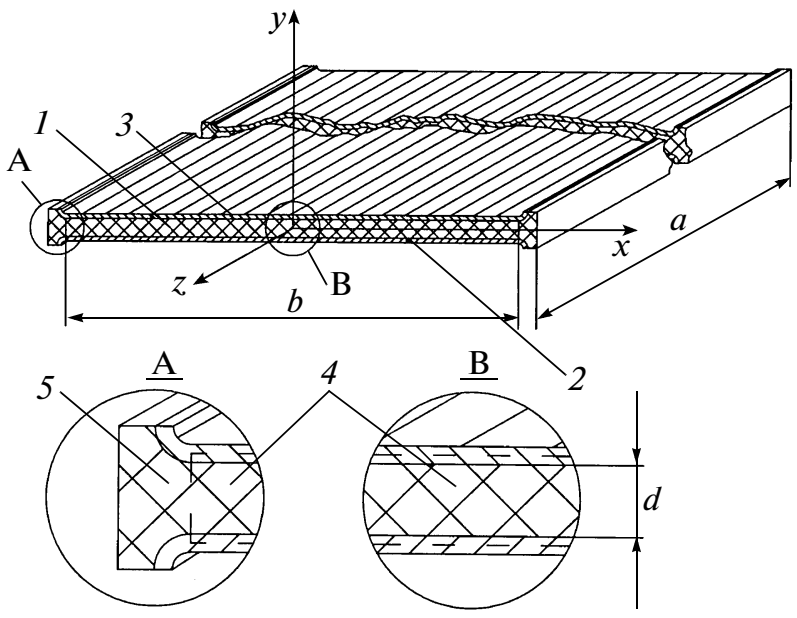

Fig. 1. Schematic diagram of the forming line (FL) in which electromagnetic wave propagation is simulated. $1-$ working medium of the line; 2, 3-earthed and potential plates, respectively; 4-computational domain (the boundaries are shown by dashed lines: $-b / 2=X_{\min }, b / 2=$ $\left.X_{\max },-d / 2=Y_{\min }, d / 2=Y_{\max },-a=Z_{\min }, Z_{\max }=0\right) ; 5-$ the edge zone; $d$ is the thickness of the dielectric; $a$ is the FL length, and $b$ is the FL width. 


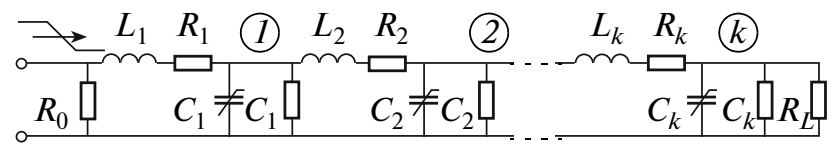

Fig. 2. Equivalent electric circuit of the forming line.

simulation make it possible to give recommendations concerning the required nonlinearity of the permittivity of the working medium, as well as its geometric configuration.

It is possible to study transient processes in FL by presenting it in the form of an equivalent electric circuit consisting of a set of in-parallel capacitances $\left(C_{1}-\right.$ $C_{k}$, Fig. 2) and series-connected inductances $\left(L_{1}-L_{k}\right.$, Fig. 2), the so-called long line with the lumped parameters or artificial extension line (see, for example $[6,7]$ ). Such an approach is possible because the electromagnetic processes in this circuit are described by the equations similar to those that describe field processes in double-wire long lines (see, for example [1,6]). Another possible solution is the computation of the electromagnetic field during wave propagation. Finally, physical simulation of the processes being investigated can be performed by measuring currents and voltages in the corresponding electric circuit.

The aim of this work is physical and mathematical simulation of electromagnetic wave sharpening in a dielectric with nonlinear parameters in order to determine the extent of influence of the main factors, as well as comparison of the results obtained with the help of different models.

\section{FORMULATION OF THE COMPUTATIONAL PROBLEM FOR THE TRANSIENT PROCESS OF ELECTROMAGNETIC WAVE PROPAGATION IN A NONLINEAR DIELECTRIC}

To analyze the process of propagation of an electromagnetic wave with a short front (on the order of units or tens of nanoseconds), the problem must be formulated taking into account the dielectric viscosity of the working medium. For the solution we use the socalled modified vector magnetic potential A (see, for example $[8,9])$. Such an approach reduces substantially the number of solvable equations. In this case, the scalar electric potential is assumed to be zero (see, for example [10-14]). Then, electric field strength $\mathbf{E}$ and magnetic field induction $\mathbf{B}$ can be expressed in terms of $\mathbf{A}$ :

$$
\begin{aligned}
& \mathbf{B}=\operatorname{curl} \mathbf{A} ; \\
& \mathbf{E}=-\frac{\partial \mathbf{A}}{\partial t} .
\end{aligned}
$$

Due to nonlinear dependence of the relative permittivity of the working medium (ferroelectric) on the electric field, as well as the necessary allowance for

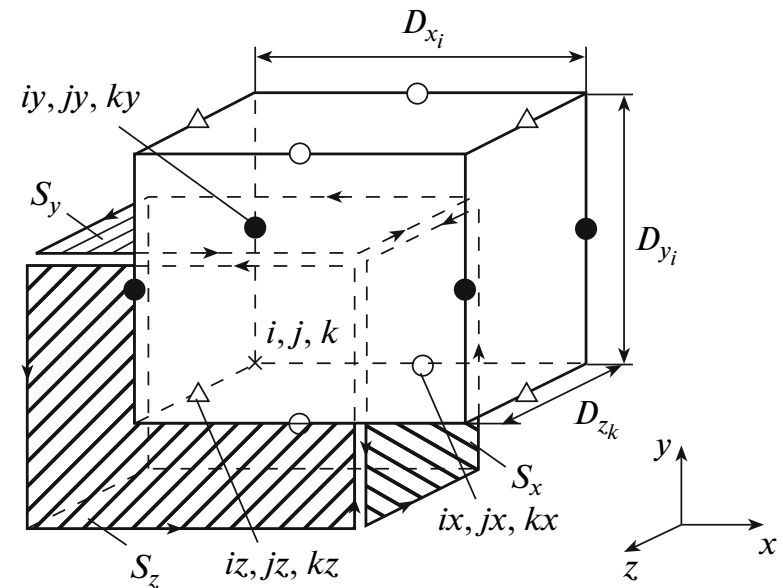

Fig. 3. A cell of the design diagram. Quantities $i x, i y, k z$, and so on are the identifiers which correspond to the numbers of points in which the values of the components of vector potential A are determined.

dielectric viscosity (i.e., time dependence) $\varepsilon(E, t)$, analytical solution of the formulated problem seems impossible; therefore, numerical methods should be applied.

It is well known that the electric field strength increases at the FL edges. To reduce this effect special techniques are conventionally used (for example, a smooth increase in the insulation thickness at the edges; see 5 in Fig. 1). In the case under consideration, the line width exceeds the dielectric thickness by an order of magnitude and more; consequently, it means that a fraction of the area of the edge zone in the total area of the zone under the plates is not large. In order to study the influence of the main factors on the electromagnetic wave sharpening during its propagation along the FL, we will simulate this process without taking into account the edges; the effect of the FL edges will be discussed separately.

To solve the problem of electromagnetic field computation, we used a numerical method of finite volumes, also called the finite integration technique (see, for example $[15,16]$ ). This method assumes the application of a computational grid on the domain under consideration and integration of the Maxwell equations over the volumes or faces of the formed cells. Such an approach makes it possible to obtain solvable equations using the conservation laws (for charge or current, respectively). Therefore, it is not necessary to introduce additional conditions at the interfaces between the media since they are automatically taken into account. This technique also makes it possible to take into account nonlinearity of electric parameters of the media in computing the electromagnetic field distribution.

To find the electromagnetic field distribution, we partition the domain under consideration into cells (parallelepipeds; Fig. 3). This partition is made so that 
the computational grid points $(i, j, k)$ lie on the interfaces between the media. The $(i, j, k)$ th cell is a parallelepiped with the following points as the vertices: $(i, j, k)$, $(i+1, j, k),(i, j+1, k),(i, j, k+1),(i, j+1, k+1)$, $(i+1, j, k+1),(i+1, j+1, k)$, and $(i+1, j+1, k+1)$. The electric parameters of the medium are assumed to be identical within each cell. Identifiers $i x, j y, k z$, etc., correspond to the numbers of the points at which the values of the components of vector potential $\mathbf{A}$ are determined. Thus, for example, the $x$ th component of $\mathbf{A}\left(A_{x}\right)$ is determined in points $i x, j x$, and $k x$.

We obtain a solvable equation by integrating the first Maxwell equation

$$
\operatorname{curl} \mathbf{H}=\gamma \mathbf{E}+\frac{\partial \mathbf{D}}{\partial t},
$$

where $\mathbf{H}$ is the magnetic field strength, $\mathbf{D}$ is the electric field induction, and $\gamma$ is the electrical conductivity along each of the three cross sections perpendicular to the coordinate axes. For example, the equation for $A_{z}$, which is the $z$ th component of vector $\mathbf{A}$, we obtain by integrating Eq. (3) over cross section $S_{z}(z=$ const, see Fig. 3) and using the Stokes theorem:

$$
\oint_{l_{z}} \mathbf{H} d \mathbf{l}=\int_{S_{z}} \gamma E_{n} d s+\int_{S_{z}} \frac{\partial D_{n}}{\partial t} d s,
$$

where $l_{z}$ is the contour embracing area element $S_{z}$ and $E_{n}$ is the projection of vector $\mathbf{E}$ on the normal to plane $S_{z}$.

In order to reduce the order of equations in form (4), these equations were integrated with respect to time:

$$
\int_{0 l_{z}}^{t} \oint_{l_{z}} \mathbf{H} d \mathbf{l} d t=\int_{0}^{t} \int_{S_{z}} \gamma E_{n} d s d t+\int_{0}^{t} \frac{\partial}{\partial t} \int_{S_{z}} D_{n} d s d t .
$$

Expressing $\mathbf{H}$ and $\mathbf{E}$ in terms of $\mathbf{A}$ (see Eqs. (1) and (2)) and taking into account zero initial conditions, for instant $t$ we finally get

$$
\frac{1}{\mu_{0}} \int_{0}^{t} \oint_{l_{z}} \frac{1}{\mu} \operatorname{curl} \mathbf{A} d \mathbf{l} d t=\int_{S_{z}}-\gamma A_{z} d s+\varepsilon_{0} \int_{S_{z}}-\varepsilon \frac{\partial A_{z}}{\partial t} d s,
$$

where $\mu$ and $\varepsilon$ are the relative permeability and permittivity, which may depend on field strengths and time; $\mu_{0}=4 \pi \times 10^{-7} \mathrm{H} / \mathrm{m}$, and $\varepsilon_{0}=0.885 \times 10^{-11} \mathrm{~F} / \mathrm{m}$.

Let us write the equations of form (6) for the remaining components of vector $\mathbf{A}$, integrating Eq. (4) over the corresponding area elements (see Fig. 3), $S_{x}$ for $A_{x}$ and $S_{y}$ for $A_{y}$ :

$$
\begin{gathered}
\frac{1}{\mu_{0}} \int_{0}^{t} \oint_{l_{x}} \frac{1}{\mu} \operatorname{curl} \mathbf{A} d \mathbf{l} d t=\int_{S_{x}}-\gamma A_{x} d s+\varepsilon_{0} \int_{S_{x}}-\varepsilon \frac{\partial A_{x}}{\partial t} d s ; \\
\frac{1}{\mu_{0}} \int_{0}^{t} \frac{1}{\mu} \operatorname{curl} \mathbf{A} d \mathbf{l} d t=\int_{S_{y}}-\gamma A_{y} d s+\varepsilon_{0} \int_{S_{y}}-\varepsilon \frac{\partial A_{y}}{\partial t} d s .
\end{gathered}
$$

The conditions at the computational domain boundaries ( 4 in Fig. 1; the boundary is shown by a dashed line) in planes $X_{\min }, X_{\max }, Y_{\min }, Y_{\max }, Z_{\min }$, and $Z_{\max }$ were as follows. Since we did not take into account the increase in the electric field at the FL edges, we have $A_{x}=0, \partial A_{y} / \partial x=0$, and $\partial A_{z} / \partial x=0$ for $x=X_{\min }$ and $x=X_{\max }$. Considering that the thickness of the plates (lines 2 and 3 in Fig. 1), which are usually made from copper, exceeds significantly the skin layer thickness during the wave propagation, the currents are concentrated in thin layers on the surface of the plates. Therefore, the strengths of the electric and magnetic fields are zero outside the skin layer in planes $y=Y_{\min }$ and $y=Y_{\max }$, which means that the components of $\mathrm{A}$ are also set to be zero: $A_{x}=0, A_{y}=0$, and $A_{z}=0$. In plane $z=Z_{\min }$, we have $\partial A_{y} / \partial t=-E_{0}$ (where $E_{0}$ is the field strength of the incident electromagnetic wave), $A_{x}=0$, and $A_{z}=0$; in plane $z=Z_{\text {max }}$, we have $A_{x}=0, A_{y}=0$, and $A_{z}=0$ (short-circuit conditions were considered). The initial conditions for all vector potential components are zero.

The system of solvable equations is obtained from the expressions of form (6)-(8) written in difference form for each point of the computational mesh. The given equations were discretized by presenting the components of curlA in the form of finite differences and finding circulation curlA during the path-tracing of rectangular contours $l_{x}, l_{y}$, and $l_{z}$ embracing area elements $S_{x}, S_{y}$, and $S_{z}$ (see Fig. 3). The integrals over $S_{x}, S_{y}$, and $S_{z}$ (the terms on the right-hand side of Eqs. (6)-(8)) were assumed to be equal to the product of their area by the values of the integrand at the corresponding point, and $\varepsilon$ and $\gamma$ were averaged over the cells surrounding the point. The time integrals of the terms which contain circulation curl $\mathbf{A}$ were replaced by the sums according to the trapezium rule. The terms of these sums, containing the components of $\mathbf{A}$ from the previous time steps, were assumed to be known. As a result, the difference equations were obtained, which have the following form, for example, for the $y$ th component of the modified vector potential:

$$
\begin{gathered}
0.5 \frac{\Delta t}{\mu_{0}}\left(\Lambda_{x x} A_{y}^{m}+\sum_{m=1}^{n-1} \Lambda_{x x} A_{y}^{m}+\Lambda_{z z} A_{y}^{n}+\sum_{m=1}^{n-1} \Lambda_{z z} A_{y}^{m}\right. \\
\left.-\sum_{m=1}^{n} \Lambda_{y x} A_{x}^{m}-\sum_{m=1}^{n} \Lambda_{y z} A_{z}^{m}\right) \\
=A_{y_{i, j, k}}^{n} S G_{y_{i, j, k}}+\frac{S E_{y_{i, j, k}}^{m}\left(A_{y_{i, j, k}}^{n-1}-A_{y_{i, j, k}}^{n-1}\right)}{\Delta t},
\end{gathered}
$$

where

$$
\begin{aligned}
\Lambda_{x x} A_{y}^{n}=0.5 \sum_{i i=i-1}^{i i=i}(-1)^{i i-i+2}\left(\frac{D z_{k-1}}{\mu_{i i, j, k-1}}+\frac{D z_{k}}{\mu_{i i, j, k}}\right) \\
\times \frac{A_{y_{i i+1, j, k}}^{n}-A_{y_{i, j, k},}^{n}}{D x_{i i}}
\end{aligned}
$$

TECHNICAL PHYSICS Vol. $56 \quad$ No. 32011 


$$
\begin{aligned}
& \Lambda_{z z} A_{y}^{n}=0.5 \sum_{k k=k-1}^{k k=k}(-1)^{k k-k+2}\left(\frac{D x_{i-1}}{\mu_{i-1, j, k k}}+\frac{D x_{i}}{\mu_{i, j, k k}}\right) \\
& \times \frac{A_{y_{i, j k k+1}^{n}}^{n}-A_{y_{i, j, k k}}^{n} ;}{D z_{k k}} ; \\
& \Lambda_{y x} A_{x}^{m}=0.5 \sum_{i i=i-1}^{i i=i}(-1)^{i i-i+2}\left(\frac{D z_{k-1}}{\mu_{i i, j, k-1}}+\frac{D z_{k}}{\mu_{i i, j, k}}\right) \\
& \times \frac{A_{x_{i, j+1, k}^{m}}^{m}-A_{x_{i, j, k}}^{m}}{D y_{j}} ; \\
& \Lambda_{y z} A_{z}^{m}=0.5 \sum_{k k=k-1}^{k k=k}(-1)^{k k-k+2}\left(\frac{D x_{i-1}}{\mu_{i-1, j, k k}}+\frac{D x_{i}}{\mu_{i, j, k k}}\right) \\
& \times \frac{A_{z_{i, j+1, k k}}^{m}-A_{z_{i, j, k k}}^{m}}{D y_{j}} ; \\
& S G_{y_{i, j, k}} \\
& =0.25 \sum_{i i=i-1}^{i i=i}\left(\gamma_{i i, j, k} D x_{i i} D z_{k}+\gamma_{i i, j, k-1} D x_{i i} D z_{k-1}\right) \text {; } \\
& S E_{y_{i, j, k}}^{m} \\
& =0.25 \varepsilon_{0} \sum_{i i=i-1}^{i i=i}\left(\varepsilon_{i i, j, k}^{m} D x_{i i} D z_{k}+\varepsilon_{i i, j, k-1}^{m} D x_{i i} D z_{k-1}\right) \text {; }
\end{aligned}
$$

$D x_{i}, D y_{j}$, and $D z_{k}$ are the steps over the space in the directions of $x, y$, and $z$, respectively (see Fig. 3 ); $\mu_{i, j, k}$ and $\gamma_{i, j, k}$ are the relative permeability and electrical conductivity of the $(i, j, k)$ th cell, respectively; $\varepsilon_{i, j, k}^{m}$ is the relative permittivity of the $(i, j, k)$ th cell at instant $t_{m}$; and $A_{y_{i, j, k}}^{n}$ is the value of the $y$ th component of the vector magnetic potential in the $(i, j, k)$ th point at the $n$th instant. Double indices $i i, k k$, etc., are the summation indices.

The arrangement of the components on the leftand right-hand sides of Eq. (9) corresponds to the arrangement of the components in Eq. (8). The expressions for the remaining components of $\mathbf{A}$ were written analogously. To solve the resultant system of the difference equations, we used the algorithm of variable directions [17]. The application of this algorithm for the solution of the time-independent problem by iterative procedure is described in detail in [18].

\section{PHYSICAL AND MATHEMATICAL SIMULATION OF WAVE PROFILE VARIATION DURING ITS PROPAGATION ALONG THE LINE WITH A NONLINEAR PERMITTIVITY}

Let us represent an FL as an equivalent electric circuit containing $k$ elements, each of which consists of a series inductance and an in-parallel nonlinear capacitance (see Fig. 2). Such a representation is justified due to the similarity of the equations that describe the electromagnetic processes in the given electric circuit and in an FL consisting of potential and ground conducting plates, between which a dielectric is located (see, for example $[1,6]$ ). The equations describing the transient processes in the $k$ th element of such an equivalent circuit represent the first and second Kirchhoff laws:

$$
\begin{aligned}
& -V_{k} G_{k}-\frac{d Q_{k}}{d t}=I_{k+1}-I_{k} ; \\
& L_{k} \frac{d I_{k}}{d t}+R_{k} I_{k}=V_{k}+V_{k-1},
\end{aligned}
$$

where $L_{k}, R_{k}, C_{k}$, and $G_{k}$ are the values of the inductance, resistance, capacitance, and leakage conductance for the $k$ th element of the line; $Q_{k}=C_{k} V_{k}$ is the charge of nonlinear capacitance $C_{k} ; V_{k}$ is the voltage across nonlinear capacitance $C_{k}$; and $I_{k}$ is the current through inductance $L_{k}$.

Taking into account the dependences of the permittivity and, hence, of the capacitance, on the level of the applied voltage, we write the second term on the left-hand side of Eq. (10) in the form

$$
\begin{aligned}
\frac{d Q}{d t}= & \frac{d[C(V) V]}{d t}=\frac{d C(V)}{d V} \frac{d V}{d t} V \\
& +C(V) \frac{d V}{d t}=\frac{d V}{d t} C_{\text {eq }},
\end{aligned}
$$

where

$$
C_{d}=\frac{d C(V)}{d V} ; \quad C_{\text {eq }}=C_{d} V+C(V) .
$$

It is known that a change in the permittivity of ferroelectrics depending on the level of the electric field strength occurs with some delay, which is determined by the so-called dielectric viscosity [19]. Analogously to the magnetic viscosity [20], we present a change in the permittivity value at instant $t_{m}$ in the form

$$
\Delta \varepsilon\left(t_{m}\right)=\left[\varepsilon_{\infty}\left(E_{m}\right)-\varepsilon\left(t_{m-1}\right)\right]\left[1-\exp \left(-\frac{\Delta t}{\tau_{R}}\right)\right],
$$

where $\varepsilon_{\infty}\left(E_{m}\right)$ is the permittivity value that sets at instant $t \gg \tau_{R}(t \longrightarrow \infty)$ after the electric field with strength $E_{m}$ is applied to the dielectric; $\varepsilon\left(t_{m-1}\right)$ is the permittivity value at instant $t_{m-1}, \Delta t=t_{m}-t_{m-1}$ is the time increment, and $\tau_{R}$ is the relaxation time. Then

$$
\varepsilon\left(t_{m}\right)=\varepsilon\left(t_{m-1}\right)+\Delta \varepsilon\left(t_{m}\right) .
$$

The change in the value of capacitance $C_{\mathrm{eq}_{k}}$ (see Eq. (13)) upon the application of voltage $V_{k}\left(t_{m}\right)$ was taken into account analogously to Eqs. (14) and (15):

$$
\begin{aligned}
C_{\mathrm{eq}_{k}}\left(t_{m}\right)=C_{\mathrm{eq}_{k}}\left(t_{m-1}\right)+\left[C_{\mathrm{eq}_{\infty}}\left(V_{k}\left(t_{m}\right)\right)-C_{\mathrm{eq}_{k}}\left(t_{m-1}\right)\right] \\
\times\left[1-\exp \left(-\frac{\Delta t}{\tau_{R}}\right)\right],
\end{aligned}
$$



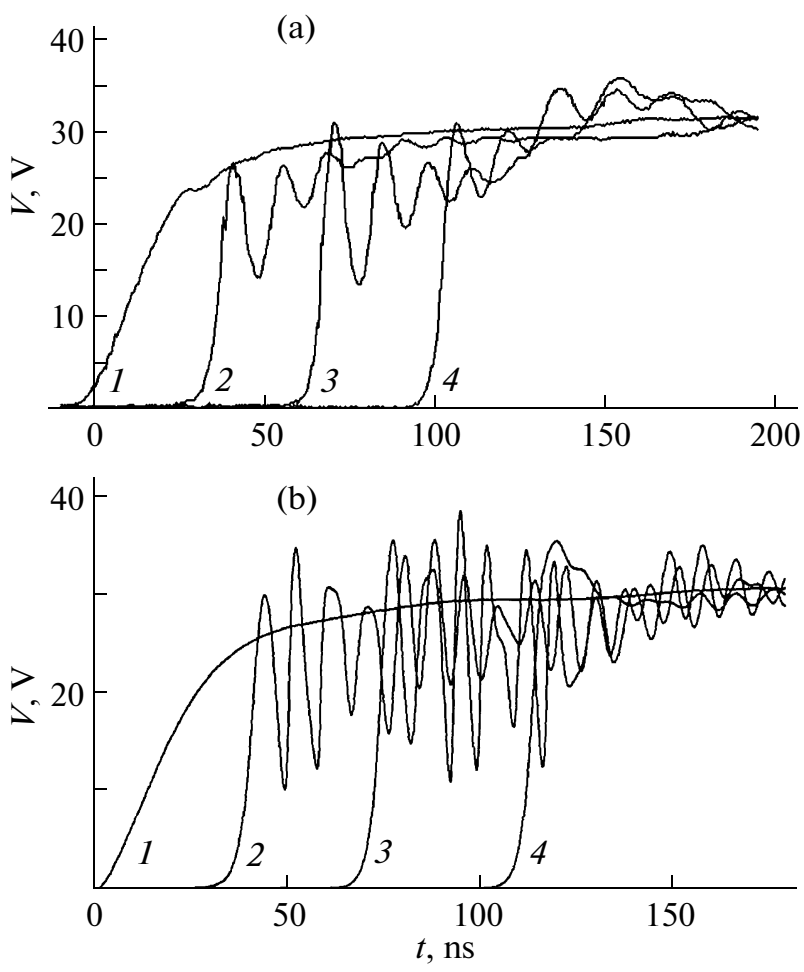

Fig. 4. Voltage time scans: 1 -at the input of the line; 2 at the 4th sharpening step; 3-at the 9th step; and 4-at the end of the line; (a) the results of physical simulation (oscillograms); (b) the results of numerical simulation based on the solution of the system of equations of form (10) and (11) with allowance for (12), (13), and (16).

where $C_{\mathrm{eq}_{\infty}}\left(V_{k}\left(t_{m}\right)\right)$ is the value of $C_{\mathrm{eq}}$ that sets at instant $t \gg \tau_{R}$ after voltage $V_{k}\left(t_{m}\right)$ has been applied.

Analyzing the process of a wave propagation in the FL, we used the value of $\tau_{R}=5 \mathrm{~ns}$, which corresponds to the dynamic characteristics applied in physical simulation of varicaps.

Time differential operators that appear in Eqs. (10) and (11) were presented in the form of differences. The resultant system of equations was solved by iterations taking into account nonlinear variation of capacitance $C_{\mathrm{eq}_{k}}$ (see Eqs. (13) and (16)). The values of currents in the circuit elements and the voltages across them at the initial instant time were set zero. We assumed that an electromagnetic wave with the given voltage $V_{0}$ is incident on the first element of the line.

For physical simulation of electromagnetic wave sharpening in the line under consideration, we assembled a circuit from $k=15$ identical $L-C$ elements (see Fig. 2) with the following parameters: $L_{k}=3.3 \mu \mathrm{H}$, $C_{0}=C_{k \mid V<0.5 \mathrm{~V}} \approx 40 \mathrm{pF}, R_{k}=1 \Omega, 1 / C_{k}=0.01 \mathrm{M} \Omega, R_{0}=$ $75 \Omega$, and $R_{L}=765 \Omega$. A rectangular pulse with amplitude $V_{0}=30 \mathrm{~V}$ and a 40 -ns-long front (at levels of $0.1 V_{0}-$ $0.9 V_{0}$ ) was applied to the line input. In this physical model, the chokes of the LQH43C series with an inductance of $3.3 \mu \mathrm{H}$ and an Ohmic resistance of $1 \Omega$ were used as inductances. BB133 varicaps served as nonlinear capacitances. The dependence of their capacitance on the applied voltage can be approximately (with a relative error not exceeding 5\%) presented in the form:

$$
C(V)[\mathrm{F}]=0.8\left[42 \exp \left(-\frac{V}{4.2}\right)+2.5\right] \times 10^{-12} .
$$

A G5-54 pulse generator connected through a cable segment with a wave impedance of $50 \Omega$ served as a source of voltage pulses applied to the input of the circuit shown in Fig. 2. The length of this cable was selected sufficiently large to maintain the output resistance of the source at the circuit input constant during the entire time of pulse propagation in the line.

Figure 4 shows the time dependences of voltage obtained experimentally (Fig. 4a), as well as from computations based on the solution of the system of nonlinear equations of form (10) and (11) (Fig. 4b). It can be seen from Fig. 4 that as the wave moves along the line, its front sharpens from 40.0 to 7.2 ns for physical simulation and to $6.6 \mathrm{~ns}$ for numerical simulation. The difference between the results of physical and capacitive simulation can probably be explained by the fact that the frequency dependences of choke parameters were not taken into account in computations.

Let us consider a variant of using the described technique of electromagnetic field computation with the help of the modified vector potential by the example of mathematical simulation of the electromagnetic wave propagation in the FL containing a ferroelectric as the working substance (see Fig. 1). As we mentioned above, the electromagnetic wave propagation was simulated without taking into account electromagnetic processes at the FL edges. In this case, we can use for comparison the solution obtained when a double-wire line along which the wave propagates was presented in the form of an electric circuit (see Fig. 2). It is easy to show that the equivalent parameters of such a circuit simulating the actual double-wire line can be computed in the following way:

$$
L_{k}=\mu_{0} \Delta z \frac{d}{b} ; \quad C_{k}=\varepsilon \varepsilon_{0} \Delta z \frac{b}{d},
$$

where $b$ is the line width; $d$ is the thickness of the dielectric (the working substance of the line); $\varepsilon$ is the permittivity of the FL working substance; $\Delta z=a / N_{z}$ is the step over the space in the direction of wave propagation; $a$ is the length of the line in the direction of wave propagation; and $N_{z}$ is the number of partitions in the direction of the $z$ axis.

Let us consider a line with the following geometric parameters: $b=50 \mathrm{~mm}, d=2 \mathrm{~mm}$, and $a=Z_{\max }=$ $0.5 \mathrm{~m}$, which operates under short-circuit conditions $\left(R_{L}=0\right.$, Fig. 2). We select the number $N_{z}$ of line elements to be equal to $350 ;\left.\varepsilon\right|_{E \rightarrow 0}=8500$. Then, the inductance and capacitance of the line, computed according to Eq. (17), are $L_{k}=0.1 \mathrm{nH}$ and $\left.C_{k}\right|_{E \rightarrow 0}=$ $3.76 \mathrm{nF}$. The leakage conductance through the dielec- 


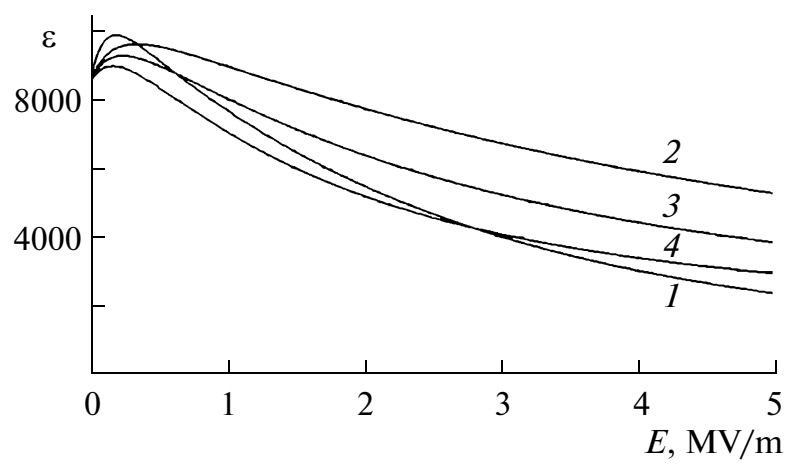

Fig. 5. Permittivity as a function of the electric field strength. 1-relative differential permittivity; $2-4$-relative permittivity; $k_{\varepsilon}=1.1$ (2); 1.34 (3); and 1.52 (4).

tric and the Ohmic resistance of the plates will be assumed to be negligibly small. When simulating the electric properties of the working substance, we used the electric parameters of ferroelectric ceramics based on barium and strontium titanates $\left(\mathrm{Ba}_{0.8} \mathrm{Sr}_{0.2} \mathrm{TiO}_{3}\right)$ solid solution doped with zirconium at the Curie temperature. The experimentally obtained dependence of relative differential permittivity $\varepsilon_{d}=\left(1 / \varepsilon_{0}\right)(\partial D / \partial E)$ on the electric field strength $|\mathbf{E}|$ for the given composition is shown in Fig. 5 (see curve 1). By integrating this curve over the electric field, we obtained the dependence of a relative permittivity on $|\mathbf{E}|$ :

$$
\varepsilon=(1 / E) \int \varepsilon_{d} d E,
$$

which is also shown in Fig. 5 (curve 2). The effect of dielectric viscosity was taken into account by using Eqs. (14) and (15) for computing $\varepsilon(t)$. The computation was carried out with relaxation time $\tau_{R}=10 \mathrm{~ns}$, which corresponds to the dynamic characteristics of the ferroelectric being considered.

Let us analyze the process of propagation of a rectangular electromagnetic wave with a rising front with a length of $80 \mathrm{~ns}$ over voltage levels of $0.1 V_{0}-0.9 V_{0}$ along the line with the described parameters. Figure 6 shows the computed time dependences of relative value of voltage $V / V_{0}$ (where $V_{0}$ is the incident wave voltage) across different sections of FL: $z=0$ (curves 1 ) and $0.25 Z_{\max }(2)$ at the beginning of FL, $z=0.5 Z_{\max }$ (3) in the middle, and $z=0.75 Z_{\max }(4)$ at the end of the line. The computations were carried out by solving circuit (Eqs. (10) and (11)) and field (Eqs. (6)-(8)) problems. A comparison of the results of such simulation shows that they coincide within a relative error not exceeding $1 \%$. The dependences shown in Fig. 6a correspond to different levels of the applied voltage: $V_{0}=$ $4 \mathrm{kV}$ (curves $2^{\prime}$ ), $V_{0}=6 \mathrm{kV}\left(3^{\prime}\right)$, and $V_{0}=8 \mathrm{kV}\left(4^{\prime}\right)$. It is clear from Fig. $6 \mathrm{a}$ that as the electromagnetic wave propagates along the line, its front sharpens. For example, the front length determined from levels $0.1 V_{0}-0.9 V_{0}$ changes from $\tau_{F 0}=80 \mathrm{~ns}$ at the beginning to $\tau_{F L}=54 \mathrm{~ns}$ at the end of the line when a voltage with an amplitude of $V_{0}=4 \mathrm{kV}$ is applied. When $V_{0}=6 \mathrm{kV}$
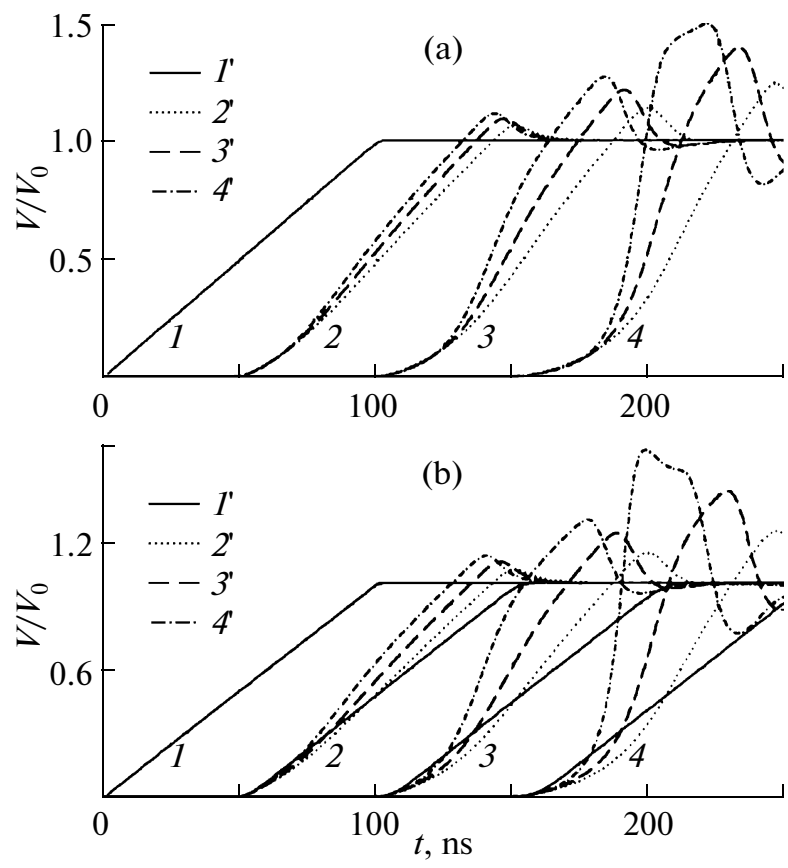

Fig. 6. Computed time dependences of the voltage at different FL cross sections: $Z=0(1) ; 0.25 Z_{\max }(2) ; 0.50 Z_{\max }$ (3); $0.75 Z_{\max }$ (4) (a) at different voltages of the incident wave $V_{0}, \mathrm{kV}: 4\left(2^{\prime}\right) ; 6\left(3^{\prime}\right)$; and $8(4)$; (b) at different slopes of the $\varepsilon(E)$ dependence: $\varepsilon$ is independent of $E\left(l^{\prime}\right) ; k_{\varepsilon}=$ $0.90\left(2^{\prime}\right) ; 0.75\left(3^{\prime}\right)$; and $0.66\left(4^{\prime}\right)$.

and $8 \mathrm{kV}$ are applied, the computed value of $\tau_{F L}$ are 39 and $22 \mathrm{~ns}$, respectively.

We also analyzed the effect of the sleepness of curve $\varepsilon(E)$ on the pulse front sharpening during the propagation along an FL with a nonlinear permittivity. To this end, we used curves $\varepsilon(E)$ similar to that given in Fig. 5 (curve 2), but with larger values of the gradient of slope (see Fig. 5, curves 3, 4), which corresponds to different temperature conditions of operation of a nonlinear dielectric. We introduce coefficient $k_{\varepsilon}=\varepsilon\left(E_{0}\right) / \varepsilon(0)$, which describes the difference of values of $\varepsilon$ in the case when the permittivity is maximal, as well as the case when the following fields are applied at the FL input: $E_{0}=V_{0} / d=2 \mathrm{MV} / \mathrm{m}\left(V_{0}=4 \mathrm{kV}, d=2 \mathrm{~mm}\right)$. Curves 2 , 3 , and 4 in Fig. 5 corresponds to $k_{\varepsilon}=0.90,0.75$, and 0.66 , respectively. Figure $6 \mathrm{~b}$ shows the computed time dependences of $V / V_{0}$ at the FL cross sections $z=$ const when voltage $V_{0}=4 \mathrm{kV}$ is applied. The curves in Fig. $6 \mathrm{~b}$ correspond to different degrees of nonlinearity of the ferroelectric (working substance of the line): $k_{\varepsilon}=0.90$ (curves $2^{\prime}$ ), $0.75\left(3^{\prime}\right)$, and $0.66\left(4^{\prime}\right)$. Curves $l^{\prime}$ correspond to the case when $\varepsilon$ is independent of $E$. Analysis of electromagnetic processes occurring during the propagation of a wave along the FL with the parameters described above shows that its front sharpens from $\tau_{F 0}=80 \mathrm{~ns}$ to $\tau_{F L}=54$ ns at $k_{\varepsilon}=0.9 ; \tau_{F L}=37$ ns at $k_{\varepsilon}=$ 0.75 ; and $\tau_{F L}=16.7 \mathrm{~ns}$ at $k_{\varepsilon}=0.66$ ns (see Fig. 6b).

Comparison of the curves in Fig. 6 shows the effectiveness of using ferroelectrics with the maximum pos- 
sible degree of nonlinearity as the FL working substance. Such a method of producing steep fronts of electromagnetic waves is more advantageous than an increase in the electric field strength, since the latter method requires the functioning of the FL at higher voltages. If coefficient $k_{\varepsilon}$, which characterizes the ratio between minimum and maximum levels of a relative permittivity, decreases from 0.90 to 0.66 , the degree of sharpening of wavefront $K_{w}=\tau_{F 0} / \tau_{F L}$ increases from 1.48 to 4.80 at the same voltage of the incident wave (see Fig. 6b). At the same time, the increase in the applied voltage from $V_{0}=4$ to $8 \mathrm{kV}$ results in an increase in $K_{w}$ from 1.48 to only 3.6 (see Fig. 6a).

\section{CONCLUSIONS}

Using physical and mathematical simulation, we have investigated the electromagnetic wavefront sharpening during the wave propagation along the forming line with a nonlinear permittivity of the working substance. We have proved that the proposed mathematical models are valid for describing the processes involved. Comparison of the data obtained from analysis of the transient process in the equivalent electric circuit with nonlinear capacitances and the results of physical simulation shows that the front durations coincide to within $10 \%$. We have shown that the modified vector magnetic potential can be used for simulation of the electromagnetic wave propagation in a nonlinear dielectric. The data obtained by solving the circuit and field problems for the case when the effects at the FL edges can be neglected coincide to within $1 \%$. With the help of numerical simulation, we have analyzed the influence of the wave amplitude and degree of nonlinearity of the dielectric on the wavefront sharpening. Thus, for example, the twofold increase in the voltage of the incident wave results in a reduction in the front duration by a factor of 2.4 , while an increase in the difference in $\varepsilon$ by 1.38 times results in a reduction in the front duration by a factor of 3.2.

\section{REFERENCES}

1. G. A. Mesyats, Pulsed Power and Electronics (Nauka, Moscow, 2004) [in Russian].
2. I. G. Katayev, Electromagnetic Shock Waves (Sovetskoe Radio, Moscow, 1963; Iliffe, London, 1966).

3. Yu. K. Bogatyrev, Izv. Vyssh. Uchebn. Zaved., Radiofiz., No. 8, 1171 (1965).

4. A. V. Gaponov and G. I. Freidman, Zh. Eksp. Teor. Fiz. 36, 957 (1959).

5. T. N. Verbitskaya, Variconds (Gosenergoizdat, Moscow, 1958) [in Russian].

6. G. Branch and P. W. Smith, J. Phys. 29, 2170 (1996).

7. M. I. Finkel'shtein, Artificial Lines (Voennoe Izd., Moscow, 1961).

8. O. Biro and K. Preis, IEEE Trans. Magn. 25, 3145 (1989).

9. M. Clemens and T. Weiland, IEEE Trans. Magn. 39, 1175 (2003).

10. J. D. Jackson, Classical Electrodynamics (Wiley, New York, 1975; Inostrannaya Literatura, Moscow, 1965).

11. N. S. Koshlyakov, E. B. Gliner, and M. M. Smirnov, Differential Equations of Mathematical Physics (Vysshaya Shkola, Moscow, 1970; North-Holland, Amsterdam, 1964).

12. J. A. Stratton, Electromagnetic Theory (McGraw-Hill, New York, 1941; Gostekhizdat, Moscow-Leningrad, 1948).

13. W. R. Smythe, Static and Dynamic Electricity (McGraw-Hill, New York, 1950; Inostrannaya Literatura, Moscow, 1954).

14. P. M. Morse and H. Feshbach, Methods of Theoretical Physics (McGraw-Hill, New York, 1953; Inostrannaya Literatura, Moscow, 1959), Vol. 1.

15. M. Clemens and T. Weiland, IEEE Trans. Magn. 38, 569 (2002).

16. S. Patankar, Numerical Heat Transfer and Fluid Flow (McGraw-Hill, New York, 1980; Energoatomizdat, Moscow, 1984).

17. A. A. Samarskii, The Theory of Difference Schemes (Nauka, Moscow, 1989; Dekker, New York, 2001).

18. M. M. Rezinkina, Zh. Tekh. Fiz. 77 (11), 17 (2007) [Tech. Phys. 52, 1407 (2007)].

19. S. A. Sadykov, V. Z. Borodin, and A. Sh. Agalarov, Zh. Tekh. Fiz. 70 (6), 108 (2000) [Tech. Phys. 45, 776 (2000)].

20. Physical Encyclopedia, http://www.femto.com.ua/articles/part_1/2039.html.

Translated by N. Wadhwa 